\title{
Oral Cancer Knowledge and Awareness in Patients Visiting Kantipur Dental College
}

\author{
Dipshikha Bajracharya, ${ }^{1}$ Sujaya Gupta, ${ }^{2}$ Manish Sapkota, ${ }^{3}$ Shishir Bhatta ${ }^{3}$ \\ ${ }^{1}$ Department of Oral and Maxillofacial Pathology, ${ }^{2}$ Department of Periodontics, ${ }^{3}$ Department of Oral and \\ Maxillofacial Pathology, Kantipur Dental College, Teaching Hospital and Research Center, Kathmandu, \\ Nepal.
}

\section{ABSTRACT}

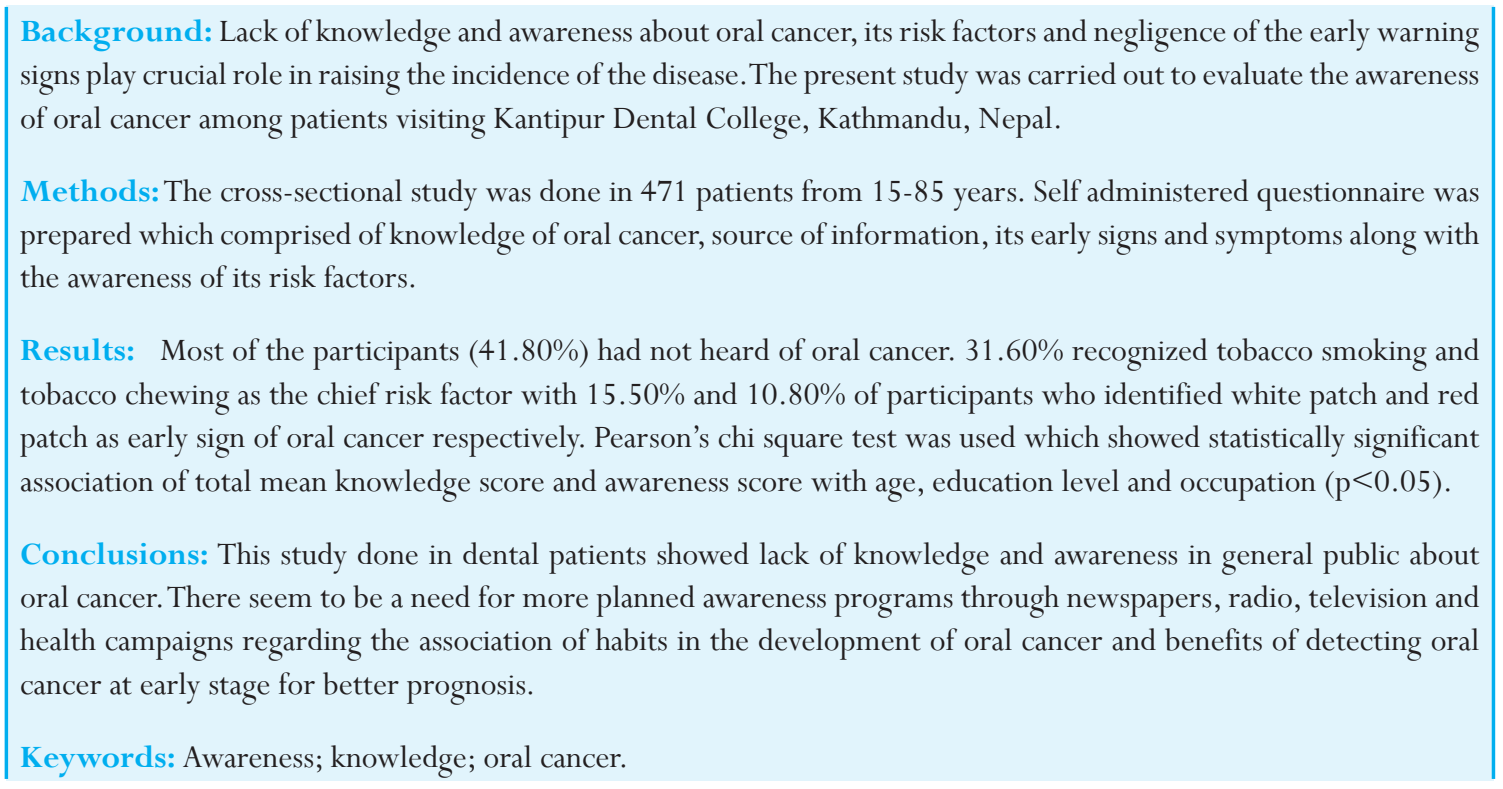

\section{INTRODUCTION}

Globally, oral cancer is a serious and life threatening problem. The annual estimated incidence of which is around 275,000 in developing countries with the highest incidence in Southeast Asia. ${ }^{1}$ Nepal does not have any well documented data regarding the frequency of oral cancer. However, a multi-institutional hospital based study has shown cancer of oral cavity to be the second most common in males. ${ }^{2}$ Oral cancer has a direct relationship with oral habits. Moreover, inadequate knowledge about the disease and lack of awareness about its risk factors play vital role in increasing incidence of oral cancer. ${ }^{1,3}$ The aim of this study was to assess the level of knowledge and awareness about oral cancer among the patients visiting Kantipur Dental College.

\section{METHODS}

A cross-sectional study was performed in the outpatient department of Kantipur Dental College and Hospital Kathmandu, Nepal from May 2016 to Feb 2017.
Participants from 15-85 years with their written consent were considered for the study. Institutional Research committee (IRC) approval was taken before carrying on the study.

A structured questionnaire was developed from the previously validated items ${ }^{4-7}$ with few modifications to suit the population and 18 closed-ended questions comprised of knowledge of oral cancer, source of information, its early signs and symptoms along with the awareness of its risk factors and the various cancers occurring in other anatomical sites. Out of which, seven were used for knowledge score, 10 for awareness score and one for source of information. The subjects were assessed based upon yes, no or don't know response. The knowledge as well as the awareness score was calculated wherein the correct response was given score of ' 1 ' while the "incorrect response" or "don't know” response was given score of ' 0 '. ${ }^{7}$ Sociodemographic data comprised of age, sex, education level and occupation. The education level was grouped as Illiterate, Primary (up to grade Correspondence: Dr Dipshikha Bajracharya, Department of Oral and Maxillofacial Pathology, Kantipur Dental College, Teaching Hospital and Research Center, Kathmandu, Nepal. Email: drdipshikhabaj@gmail.com, Phone: +9779860536501. 
5), Lower secondary (up to grade 8 ), Higher secondary (up to grade 10) and college/ university. Likewise, the occupation was categorized as Professionals, Service, Skilled workers, Laborers and related, Student, Trades and related, Unemployed and Retired.

Statistical package for social science (SPSS) version 20.0 was used for the data entry and analysis. The data were presented as means and standard deviations and the outcome were analyzed using Pearson's Chi-square test. $p$-value was calculated under the predetermined level of significance of 0.05 at the confidence level of $95 \%$.

\section{RESULTS}

In the present study, Table 1 represents the sociodemographic characteristics. A total of 471 subjects participated in the study, among which only $182(38.60 \%)$ had heard about oral cancer, whereas approximately half of the subjects had no idea about it $289(41.80 \%)$. Among the subjects who had heard of oral cancer, $30.10 \%$ had gained information from mass media, $10.80 \%$ from Dentists as well as Friends and family, 5.30\% from Health camps and $4.70 \%$ from Physicians. The study sample comprised of almost equal distribution of Male (49.70\%) and Female $(50.30 \%)$ with no significant gender difference in the knowledge as well as awareness score about the existence of oral cancer (Table 2 and Table 3 ). The age of participants in the study were ranging from 17 to 83 years with the mean $34.71 \pm 13.3$ years (Table 1 ).

Table 1. Frequency distribution of the

Sociodemographic characteristics.

\begin{tabular}{|c|c|c|c|}
\hline Characteristics & Categories & $\begin{array}{r}\text { Frequency } \\
\text { (n) }\end{array}$ & $\begin{array}{r}\text { Percent } \\
(\%)\end{array}$ \\
\hline Total & & 471 & 100 \\
\hline \multirow[t]{2}{*}{ Gender } & Male & 234 & 49.7 \\
\hline & Female & 237 & 50.3 \\
\hline \multirow[t]{6}{*}{ Age } & $\leq 20$ & 63 & 13.37 \\
\hline & $21-35$ & 204 & 43.31 \\
\hline & $36-50$ & 141 & 29.93 \\
\hline & $51-65$ & 52 & 11.04 \\
\hline & $66-80$ & 10 & 2.12 \\
\hline & $>80$ & 1 & 0.21 \\
\hline \multirow[t]{6}{*}{ Education } & No response & 4 & 0.84 \\
\hline & Illiterate & 26 & 5.52 \\
\hline & Primary & 32 & 6.79 \\
\hline & Lower Sec & 25 & 5.30 \\
\hline & Higher Sec & 132 & 28.02 \\
\hline & $\begin{array}{l}\text { College/ } \\
\text { University }\end{array}$ & 252 & 53.50 \\
\hline Occupation & No response & 97 & 20.59 \\
\hline
\end{tabular}

\begin{tabular}{|lrr|}
\hline Professionals & 25 & 5.30 \\
\hline Service & 55 & 11.67 \\
\hline $\begin{array}{l}\text { Skilled } \\
\text { workers, } \\
\text { laborers \& } \\
\text { related }\end{array}$ & 39 & 8.28 \\
\hline $\begin{array}{l}\text { Student } \\
\text { Trades \& } \\
\text { related }\end{array}$ & 129 & 27.38 \\
\hline $\begin{array}{l}\text { Unemployed } \\
\text { \& Retired }\end{array}$ & 68 & 14.43 \\
\hline
\end{tabular}

Half of the study population had college/university education with the study participants mostly comprising of students (Table 1). In the present study, only about $29.10 \%$ of the subjects believed that oral cancer was preventable while more than half of the participants $63.90 \%$ had no idea whether it was preventable or not. Likewise, among the study participants, only $22.30 \%$ believed that oral cancer was not contagious with $5.70 \%$ (Table 2) who believed it was contagious further indicating the low knowledge among the study subjects regarding oral cancer.

The subjects showed slight awareness about the risk factors of oral cancer with $31.60 \%$ who recognized tobacco-smoking and tobacco-chewing as the chief risk factor. Alcohol drinking was believed to be the sole risk factor by $17.80 \%$ whereas betel-quid chewing was considered as risk factor by $12.30 \%$ with $4.20 \%$ participants who thought old age as a risk factor in causing oral cancer (Table 3 ).

The subjects showed poor knowledge regarding the early signs and symptoms of oral cancer with only $15.50 \%$ and $10.80 \%$ of the total, who identified white patch and red patch as early sign respectively. Similarly, only about $10 \%$ of the participants identified unhealed ulcer as early sign of oral cancer. Around $23.10 \%$ and $11.50 \%$ of the participants had misconception that recurrent dental abscess and dental caries were the early signs of oral cancer respectively (Table 2 ). The study showed that more than half of the total subjects were aware about Lung cancer (64.33\%) followed by Cervix (50.70\%), Breast (45.20\%), Oral (36.10\%), Gastro-intestinal (GI) (21.90\%) and other cancers (4.50\%) (Table 3$)$.

The total mean knowledge score was $1.26 \pm 1.76$ (range $0-7)$ with significant association $(p<0.05)$ with age, education and occupation (Table 2). Likewise, the total mean awareness score was 3.96 \pm 2.75 (range $0-10$ ) having significant $(\mathrm{p}<0.05)$ association with age, education level and occupation (Table 3). 
Oral Cancer Knowledge and Awareness in Patients Visiting Kantipur Dental College

\begin{tabular}{|c|c|c|c|c|c|c|c|c|}
\hline \multicolumn{2}{|c|}{$\begin{array}{l}\text { Sociodemographic } \\
\text { characteristics }\end{array}$} & \multirow{2}{*}{$\begin{array}{r}\begin{array}{r}\text { Knowledge } \\
\text { of oral } \\
\text { cancer }\end{array} \\
\text { n (\%) } \\
92(19.53)\end{array}$} & \multirow{2}{*}{$\begin{array}{r}\text { Do you } \\
\text { think oral } \\
\text { cancer is } \\
\text { preventable } \\
n(\%) \\
61(12.95)\end{array}$} & \multirow{2}{*}{$\begin{array}{r}\text { Do you } \\
\text { think oral } \\
\text { cancer is } \\
\text { contagious } \\
n(\%) \\
13(2.76)\end{array}$} & \multirow{2}{*}{$\begin{array}{r}\text { Lump } \\
\text { as sign } \\
\text { of oral } \\
\text { cancer } \\
\mathrm{n}(\%)\end{array}$} & \multirow{2}{*}{$\begin{array}{r}\text { White } \\
\text { patch } \\
\text { as sign } \\
\text { of oral } \\
\text { cancer } \\
n(\%) \\
40(8.49)\end{array}$} & \multirow{2}{*}{$\begin{array}{r}\text { Red patch } \\
\text { as sign } \\
\text { of oral } \\
\text { cancer } \\
n(\%) \\
26(5.52)\end{array}$} & \multirow{2}{*}{$\begin{array}{r}\text { Non- } \\
\text { healing } \\
\text { ulcer as } \\
\text { sign of oral } \\
\text { cancer } n \\
(\%) \\
26(5.52)\end{array}$} \\
\hline Gender & Male & & & & & & & \\
\hline & Female & $90(19.10)$ & $76(60.13)$ & $14(2.97)$ & $40(8.49)$ & $33(7.00)$ & $25(5.34)$ & $21(4.45)$ \\
\hline \multirow[t]{6}{*}{ Age } & $\leq 20$ & $28(5.94)$ & $20(4.24)$ & $2(0.42)$ & 8 (1.69) & $9(1.91)$ & $6(1.27)$ & $2(0.42)$ \\
\hline & $21-35$ & $101(21.44)^{*}$ & $82(17.40)^{*}$ & $15(3.18)$ & $49(10.40)^{*}$ & $44(9.34)$ & $32(6.79)$ & $26(5.52)$ \\
\hline & $36-50$ & $35(7.43)$ & $24(5.09)$ & $7(1.48)$ & $13(2.76)$ & $13(2.76)$ & $7(1.48)$ & $12(2.54)$ \\
\hline & $51-65$ & $16(3.39)$ & $10(2.12)$ & $3(0.63)$ & $4(0.84)$ & $7(1.48)$ & $5(1.06)$ & $7(1.48)$ \\
\hline & $66-80$ & $2(0.42)$ & $1(0.21)$ & $0(0)$ & $1(0.21)$ & $0(0)$ & $1(0.21)$ & $0(0)$ \\
\hline & $>80$ & $0(0)$ & $0(0)$ & $0(0)$ & $0(0)$ & $0(0)$ & $0(0)$ & $0(0)$ \\
\hline \multirow[t]{6}{*}{ Education } & No response & $0(0)$ & $0(0)$ & $0(0)$ & $0(0)$ & $0(0)$ & $0(0)$ & $0(0)$ \\
\hline & Illiterate & $1(0.21)$ & $1(0.21)$ & $0(0)$ & $1(0.21)$ & $0(0)$ & $0(0)$ & $0(0)$ \\
\hline & Primary & $3(0.63)$ & $2(0.42)$ & $1(0.21)$ & $1(0.21)$ & $0(0)$ & $2(0.42)$ & $1(0.21)$ \\
\hline & Lower Sec & $0(0)$ & $1(0.21)$ & $0(0)$ & $0(0)$ & $0(0)$ & $1(0.21)$ & $0(0)$ \\
\hline & Higher Sec & $19(4.03)$ & $8(1.69)$ & $6(1.27)$ & $5(1.06)$ & $4(0.84)$ & $2(0.42)$ & $5(1.06)$ \\
\hline & $\begin{array}{l}\text { College/ } \\
\text { University }\end{array}$ & $159(35.88)^{*}$ & $125(26.53)^{*}$ & $20(4.24)$ & $68(14.43)^{*}$ & $69(14.64)^{*}$ & $46(9.76)^{*}$ & $41(8.70)^{*}$ \\
\hline \multirow[t]{7}{*}{ Occupation } & No response & $10(2.12)$ & 7 (1.48) & $1(0.21)$ & $6(1.27)$ & $4(0.84)$ & $3(0.63)$ & $3(0.63)$ \\
\hline & Professionals & $24(5.09)$ & 22 (4.67) & $2(0.42)$ & $13(2.76)$ & $12(2.54)$ & 8 (1.69) & $7(1.48)$ \\
\hline & Service & $36(7.64)$ & $26(5.52)$ & 7 (1.48) & $13(2.76)$ & $19(4.03)$ & $11(2.33)$ & $13(2.76)$ \\
\hline & $\begin{array}{l}\text { Skilled } \\
\text { workers, } \\
\text { laborers \& } \\
\text { related }\end{array}$ & 9 (1.91) & 8 (1.69) & $2(0.42)$ & $4(0.84)$ & $2(0.42)$ & $2(0.42)$ & $2(0.42)$ \\
\hline & Student & $71(15.07)^{*}$ & $56(11.88)^{*}$ & 9 (1.91) & $30(10.61)^{*}$ & $26(5.52)^{*}$ & $18(3.82)^{*}$ & $11(2.33)$ \\
\hline & $\begin{array}{l}\text { Trades \& } \\
\text { related }\end{array}$ & $23(4.88)$ & $14(2.97)$ & $4(0.84)$ & 7 (1.48) & $10(2.12)$ & 8 (1.69) & $10(2.12)$ \\
\hline & $\begin{array}{l}\text { Unemployed } \\
\text { \& retired }\end{array}$ & 9 (1.91) & $4(0.84)$ & $2(0.42)$ & $2(0.42)$ & $0(0)$ & $1(0.21)$ & $1(0.21)$ \\
\hline
\end{tabular}

$$
*: p<0.05
$$

\begin{tabular}{|c|c|c|c|c|c|c|c|c|c|c|c|}
\hline \multicolumn{2}{|c|}{$\begin{array}{l}\text { Sociodemographic } \\
\text { characteristics }\end{array}$} & $\begin{array}{r}\text { Awareness } \\
\text { about any } \\
\text { cancer } \\
\text { n (\%) }\end{array}$ & $\begin{array}{r}\text { Tobacco } \\
\text { smoking } \\
\text { n (\%) }\end{array}$ & $\begin{array}{r}\text { Tobacco } \\
\text { chewing } \\
\text { n (\%) }\end{array}$ & $\begin{array}{r}\text { Betel } \\
\text { quid } \\
\text { chewing } \\
n(\%)\end{array}$ & $\begin{array}{r}\text { Alcohol } \\
\text { consum } \\
\text { ption } \\
\\
\mathrm{n}(\%)\end{array}$ & $\begin{array}{l}\text { Lungs } \\
\text { n (\%) }\end{array}$ & $\begin{array}{r}\mathrm{Gl} \\
\mathrm{n}(\%)\end{array}$ & $\begin{array}{l}\text { Oral } \\
\text { n (\%) }\end{array}$ & $\begin{array}{l}\text { Breast } \\
\mathrm{n}(\%)\end{array}$ & $\begin{array}{r}\text { Cervix } \\
\mathrm{n}(\%)\end{array}$ \\
\hline \multirow[t]{2}{*}{ Gender } & Male & $\begin{array}{r}214 \\
(45.43)\end{array}$ & $\begin{array}{r}81 \\
(17.19)\end{array}$ & $\begin{array}{r}76 \\
(16.13)\end{array}$ & $\begin{array}{r}28 \\
(5.94)\end{array}$ & $\begin{array}{r}49 \\
(10.40)\end{array}$ & $\begin{array}{r}184 \\
(39.06)\end{array}$ & $\begin{array}{r}67 \\
(14.22)\end{array}$ & $\begin{array}{r}87 \\
(18.47)\end{array}$ & $\begin{array}{r}87 \\
(18.47)\end{array}$ & $\begin{array}{r}87 \\
(18.47)\end{array}$ \\
\hline & Female & $\begin{array}{r}210 \\
(44.58)\end{array}$ & $\begin{array}{r}68 \\
(14.43)\end{array}$ & $\begin{array}{r}73 \\
(15.49)\end{array}$ & $\begin{array}{r}30 \\
(6.36)\end{array}$ & $35(7.43)$ & $\begin{array}{r}119 \\
(25.26)\end{array}$ & $\begin{array}{r}36 \\
(7.64)\end{array}$ & $\begin{array}{r}83 \\
(17.62)\end{array}$ & $\begin{array}{r}126 \\
(26.75)\end{array}$ & $\begin{array}{r}152 \\
(32.27)\end{array}$ \\
\hline \multirow[t]{6}{*}{ Age } & $\leq 20$ & $62(13.16)$ & $\begin{array}{r}18 \\
(3.82)\end{array}$ & $\begin{array}{r}22 \\
(4.67)\end{array}$ & $8(1.69)$ & $7(1.48)$ & $\begin{array}{r}39 \\
(8.28)\end{array}$ & $8(1.69)$ & $\begin{array}{r}23(4 . \\
88)\end{array}$ & $\begin{array}{r}35 \\
(7.43)\end{array}$ & $\begin{array}{r}35 \\
(7.43)\end{array}$ \\
\hline & $21-35$ & $\begin{array}{r}187 \\
(39.70)\end{array}$ & $\begin{array}{r}85 \\
(18.04)^{*}\end{array}$ & $\begin{array}{r}83 \\
(17.62)^{*}\end{array}$ & $\begin{array}{r}33 \\
(7.01)\end{array}$ & $46(9.76)$ & $\begin{array}{r}143 \\
(30.36)\end{array}$ & $\begin{array}{r}55 \\
(11.67)\end{array}$ & $\begin{array}{r}96 \\
(20.38)^{*}\end{array}$ & $\begin{array}{r}110 \\
(23.35)^{*}\end{array}$ & $\begin{array}{r}116 \\
(24.62)\end{array}$ \\
\hline & $36-50$ & $\begin{array}{l}121 \\
(25.69)\end{array}$ & $\begin{array}{l}30 \\
(6.36)\end{array}$ & $\begin{array}{l}27 \\
(5.73)\end{array}$ & 9 (1.91) & $\begin{array}{l}18(3 . \\
82)\end{array}$ & $\begin{array}{l}85 \\
(18.04)\end{array}$ & $\begin{array}{l}20 \\
(4.24)\end{array}$ & $\begin{array}{l}33 \\
(7.01)\end{array}$ & $\begin{array}{l}49 \\
(10.40)\end{array}$ & $\begin{array}{l}59 \\
(12.52)\end{array}$ \\
\hline & $51-65$ & $46(9.76)$ & $\begin{array}{l}15 \\
(3.18)\end{array}$ & $\begin{array}{l}16 \\
(3.39)\end{array}$ & 8 (1.69) & $12(2.54)$ & $\begin{array}{l}31 \\
(6.58)\end{array}$ & $\begin{array}{l}17 \\
(3.60)\end{array}$ & $\begin{array}{l}16 \\
(3.39)\end{array}$ & $\begin{array}{l}16 \\
(3.39)\end{array}$ & $\begin{array}{l}25 \\
(5.30)\end{array}$ \\
\hline & $66-80$ & 7 (1.48) & $1(0.21)$ & $1(0.21)$ & $0(0)$ & $1(0.21)$ & $5(1.06)$ & $3(0.63)$ & $2(0.42)$ & $3(0.63)$ & $3(0.63)$ \\
\hline & $>80$ & $1(0.21)$ & $0(0)$ & $0(0)$ & $0(0)$ & $0(0)$ & $0(0)$ & $0(0)$ & $0(0)$ & $0(0)$ & $1(0.21)$ \\
\hline
\end{tabular}


Oral Cancer Knowledge and Awareness in Patients Visiting Kantipur Dental College

\begin{tabular}{|c|c|c|c|c|c|c|c|c|c|c|c|}
\hline \multirow[t]{6}{*}{ Education } & No response & $2(0.42)$ & $1(0.21)$ & $0(0)$ & $0(0)$ & $0(0)$ & $2(0.42)$ & $0(0)$ & $0(0)$ & $1(0.21)$ & $1(0.21)$ \\
\hline & Illiterate & $18(3.82)$ & $0(0)$ & $1(0.21)$ & $0(0)$ & $1(0.21)$ & $\begin{array}{l}15 \\
(3.18)\end{array}$ & $2(0.42)$ & $1(0.21)$ & $2(0.42)$ & $3(0.63)$ \\
\hline & Primary & $23(0.49)$ & $2(0.42)$ & $1(0.21)$ & $0(0)$ & $2(0.42)$ & $\begin{array}{l}17 \\
(3.60)\end{array}$ & $\begin{array}{l}4(0 . \\
84)\end{array}$ & $2(0.42)$ & $9(1.91)$ & $\begin{array}{l}13 \\
(2.76)\end{array}$ \\
\hline & Lower Sec & $19(4.03)$ & $0(0)$ & $0(0)$ & $0(0)$ & $0(0)$ & $\begin{array}{l}11 \\
(2.33)\end{array}$ & $3(0.63)$ & $0(0)$ & $5(1.06)$ & $\begin{array}{l}10 \\
(2.12)\end{array}$ \\
\hline & Higher Sec & $\begin{array}{l}122 \\
(25.90)\end{array}$ & 183.82) & $\begin{array}{l}13 \\
(2.76)\end{array}$ & $\begin{array}{l}4(0 . \\
84)\end{array}$ & $10(2.12)$ & $\begin{array}{l}83 \\
(17.62)\end{array}$ & $\begin{array}{l}11 \\
(2.33)\end{array}$ & $\begin{array}{l}16 \\
(3.39)\end{array}$ & $\begin{array}{l}40 \\
(8.49)\end{array}$ & $\begin{array}{l}65(13 . \\
80)\end{array}$ \\
\hline & $\begin{array}{l}\text { College/ } \\
\text { University }\end{array}$ & $\begin{array}{l}240 \\
(50.95)^{*}\end{array}$ & $\begin{array}{l}128 \\
(27.17)^{*}\end{array}$ & $\begin{array}{l}134 \\
(28.45)^{*}\end{array}$ & $\begin{array}{l}54 \\
(11.46)^{*}\end{array}$ & $\begin{array}{l}71 \\
(15.07)^{*}\end{array}$ & $\begin{array}{l}175 \\
(37.15)\end{array}$ & $\begin{array}{l}83 \\
(17.62)^{*}\end{array}$ & $\begin{array}{l}151 \\
(32.05)^{*}\end{array}$ & $\begin{array}{l}156 \\
(33.12)^{*}\end{array}$ & $\begin{array}{l}147 \\
(31.21)^{*}\end{array}$ \\
\hline \multirow[t]{7}{*}{ Occupation } & No response & $71(15.07)$ & $9(1.91)$ & $\begin{array}{l}10 \\
(2.12)\end{array}$ & $\begin{array}{l}4(0 . \\
84)\end{array}$ & $7(1.48)$ & $\begin{array}{l}43 \\
(9.12)\end{array}$ & $\begin{array}{l}10 \\
(2.12)\end{array}$ & $\begin{array}{l}10 \\
(2.12)\end{array}$ & $\begin{array}{l}33 \\
(7.01)\end{array}$ & $\begin{array}{l}40 \\
(8.49)\end{array}$ \\
\hline & Professionals & $25(5.30)$ & $\begin{array}{l}17 \\
(3.60)\end{array}$ & $\begin{array}{l}24 \\
(5.09)\end{array}$ & $\begin{array}{l}11 \\
(2.33)\end{array}$ & $8(1.69)$ & $\begin{array}{l}24 \\
(5.09)\end{array}$ & $\begin{array}{l}15 \\
(3.18)\end{array}$ & $\begin{array}{l}23(4 . \\
88)\end{array}$ & $\begin{array}{l}22 \\
(4.67)\end{array}$ & $\begin{array}{l}21 \\
(4.45)\end{array}$ \\
\hline & Service & $54(11.46)$ & $\begin{array}{l}32 \\
(6.79)\end{array}$ & $\begin{array}{l}28 \\
(5.94)\end{array}$ & $\begin{array}{l}13 \\
(2.76)\end{array}$ & $21(4.45)$ & $\begin{array}{l}48 \\
(10.19)\end{array}$ & $\begin{array}{l}16 \\
(3.39)\end{array}$ & $\begin{array}{l}35 \\
(7.43)\end{array}$ & $\begin{array}{l}30 \\
(6.36)\end{array}$ & $\begin{array}{l}26 \\
(5.52)\end{array}$ \\
\hline & $\begin{array}{l}\text { Skilled } \\
\text { workers, } \\
\text { laborers \& } \\
\text { related }\end{array}$ & $34(7.21)$ & $6(1.27)$ & $5(1.06)$ & $1(0.21)$ & $4(0.84)$ & $\begin{array}{l}29 \\
(6.15)\end{array}$ & $6(1.27)$ & $7(1.48)$ & 8 (1.69) & $\begin{array}{l}14 \\
(2.97)\end{array}$ \\
\hline & Student & $\begin{array}{l}120 \\
(25.48)^{*}\end{array}$ & $\begin{array}{l}57 \\
(12.10)^{*}\end{array}$ & $\begin{array}{l}55 \\
(11.67)^{*}\end{array}$ & $\begin{array}{l}20 \\
(4.24)^{*}\end{array}$ & $\begin{array}{l}26 \\
(5.52)^{*}\end{array}$ & $\begin{array}{l}87 \\
(18.47)^{*}\end{array}$ & $\begin{array}{l}33 \\
(7.01)^{*}\end{array}$ & $\begin{array}{l}64 \\
(13.58)^{*}\end{array}$ & $\begin{array}{l}72 \\
(15.28)^{*}\end{array}$ & $\begin{array}{l}71 \\
(15.07)^{*}\end{array}$ \\
\hline & $\begin{array}{l}\text { Trades \& } \\
\text { related }\end{array}$ & $67(14.22)$ & $\begin{array}{l}20 \\
(4.24)\end{array}$ & $\begin{array}{l}19 \\
(4.03)\end{array}$ & $7(1.48)$ & $14(2.97)$ & $\begin{array}{l}52 \\
(11.04)\end{array}$ & $\begin{array}{l}18 \\
(3.82)\end{array}$ & $\begin{array}{l}23(4 . \\
88)\end{array}$ & $\begin{array}{l}25 \\
(5.30)\end{array}$ & $\begin{array}{l}28 \\
(5.94)\end{array}$ \\
\hline & $\begin{array}{l}\text { Unemployed } \\
\text { \& retired }\end{array}$ & $53(11.25)$ & $8(1.69)$ & $8(1.69)$ & $2(0.42)$ & $4(0.84)$ & $\begin{array}{l}20 \\
(4.24)\end{array}$ & $5(1.06)$ & $8(1.69)$ & $\begin{array}{l}23(4 . \\
88)\end{array}$ & $\begin{array}{l}39 \\
(8.28)\end{array}$ \\
\hline
\end{tabular}

\section{DISCUSSION}

In the present study, less than half of the study subjects had not heard of oral cancer which is similar to the results of the study done by Pakfetrat et al. (Iran), ${ }^{8}$ Sathyanarayanan et al. (India), ${ }^{9}$ Turki (Saudi Arabia). ${ }^{10}$ In a developing country such as Nepal where oral cancer accounts for the second most common cancer in middleaged male population not having heard of oral cancer by half of the study population is an alarming signal. ${ }^{2}$ The main source of information about oral cancer was found to be from mass media than other sources clearly indicating the power of media in today's world. ${ }^{5,7,11}$

We found that young subjects had more knowledge and awareness about oral cancer than older age group which is comparable to the study done by Maweri et $\mathrm{al}^{7}$ wherein there was statistically significant difference in knowledge of the participants which was attributed to the education level. The participants in our study mostly comprised of students despite which their knowledge regarding the oral cancer was low, emphasizing the need for awareness campaigns specially targeted towards younger generations. ${ }^{12}$

Subjects also showed low awareness regarding the risk habits. Though smoking and chewing tobacco were the most identified risk factors, the association of alcohol consumption with oral cancer was low which was comparable to studies done by Ghani et al. ${ }^{5}$ in Malaysian population and Dost et al. ${ }^{13}$ in Australian population. Since oral cancer has a direct relationship with habits, recognizing the risk factor is very crucial in prevention of the disease. We found that the study subjects lacked knowledge regarding the early signs and symptoms of oral cancer. Very few subjects identified unhealed ulcers as an early sign likewise red patch and white patch were believed to be sign of oral cancer by only little percentage of the subjects which denotes the lack of knowledge about the precancerous lesions in general public. Similarly, Pakfetrat et al. ${ }^{8}$ and Reddy et al. ${ }^{14}$ had also shown low knowledge regarding the early signs and symptoms of oral cancer by the participants. Less than half of the subjects believed that oral cancer was preventable as well as non contagious stating a misconception regarding oral cancer which may be directly linked to lack of knowledge towards the early changes that oral cancer can present with. In the study when "Patients were asked whether they had heard of any other cancer"? The maximum number of patient had heard about lung cancer followed by cervix and breast denoting the lack of awareness about oral cancer compared to cancer affecting other body organs which is in coherence with study done by Rogers et al. ${ }^{15}$

The present study revealed lack of knowledge and awareness towards oral cancer in general public indicating that a more structured teaching programs should be introduced right from the school level. TV, radio, newspapers should contain advertisements with 
detail information on the risk factors, harm caused by tobacco use, betel quid chewing, alcohol use along with the premalignant changes of oral cancer. ${ }^{16}$ Also our people should be motivated for a regular oral examination so that the premalignant changes can be identified and diagnosed as earliest as possible.

\section{CONCLUSIONS}

This study highlights the lack of knowledge about oral cancer in general public. Hence, people should be made aware of oral cancer through mass media, health camps and health workers. A systematic awareness programs about the role of habits in the development of oral cancer, its complication and benefits of detecting this disease at early stage needs to be implemented by the policy makers, institutions and hospitals for better patient outcome.

\section{ACKNOWLEDGEMENTS}

We would like to acknowledge Prof. Dr. S. Bhattacharyya and Kantipur Dental College for motivation and support.

\section{REFERENCES}

1. Warnakulasuriya S. Global epidemiology of oral and oropharyngeal cancer. Oral Oncol.2009;45(4-5):309-16. [Pub Med $|\underline{\text { DOI }}|$ Full text]

2. Pradhananga KK, Baral M, Shrestha BM. Multi-institution hospital-based cancer incidence data for Nepal: an initial report. Asian Pac J Cancer Prev. 2009;10(2):259-62. [Pub $\underline{\text { Med }} \mid \underline{\text { Full text] }}$

3. Warnakulasuriya K, Harris CK, Scarrott DM, Watt R, Gelbier S, Peters TJ, et al. Oral cancer: an alarming lack of public awareness towards oral cancer. Br Dent J. 1999; 187: 319-22. [ㅁub Med $\mid \underline{\text { Full text] }}$

4. Park JH, Slack-Smith L, Smith A, Frydrych AM, O’Ferrall I, Bulsara M. Knowledge and perceptions regarding oral and pharyngeal carcinoma among adult dental patients. Aus Dent J. 2011; 56: 284-9. [ [ Pub Med | DOI | Full Text]

5. Ghani WM, Doss JG, Jamaluddin M, Kamaruzaman D, Zain RB. Oral cancer awareness and its determinants among a selected Malaysian population. Asian Pac J Cancer Prev. 2013;14: 1957-63. [ub Med | Full Text]

6. Gopal KS, Duraiselvi P. Awareness and Knowledge of Oral Cancer Among Dental Patients: A Survey Based Questionnaire Study. Int J Adv Health Sci. 2014;131:12-5. [Full Text]
7. Al-Maweri SA, Addas A, Tarakji B, Abbas A, Al-Shamiri HM, Alaizari NA, Shugaa-Addin B. Public awareness and knowledge of oral cancer in Yemen. Asian Pac J Cancer Prev. 2011;15:10861-5. [ $\underline{\text { Pub Med | Full Text] }}$

8. Pakfetrat A, Falaki F, Esmaily HO, Shabestari S. Oral cancer knowledge among patients referred to Mashhad Dental School, Iran. Arch Iran Med. 2010;13(6):543- 8. [ㅁub $\underline{\text { Med }}|\underline{\text { DOI }}| \underline{\text { Full text] }}$

9. Sathyanarayanan R, Karthigeyan R, Dinesh DS. Awareness about oral cancer among Non medical university students of Puducherry. JIDENT. 2012; 1(1):1-5.[Full Text]

10. Alhazzazi TY. Evaluation of head and neck cancer awareness and screening status in Jeddah, Saudi Arabia. Asian Pac J Cancer Prev. 2016;17:1135-9. [Pub Med $\mid$ DOI | Full Text]

11. Saleh A, Yang YH, Wan Abd Ghani WM, Abdullah N, Doss JG, Navonil R, et al. Promoting oral cancer awareness and early detection using a mass media approach. Asian Pac J Cancer Prev. 2012; 13(4):1217-24. [ uㅏ Med | DOI | Full Text ]

12. Subapriya R, Thangavelu A, Mathavan B, Ramachandran $\mathrm{CR}$, Nagini S. Assessment of risk factors for oral squamous cell carcinoma in Chidambaram, Southern India: a case-control study. Eur J Can Prev. 2007;16(3):251-6. [ $\underline{\text { Pub }}$ Med ]

13. Dost F, Do L, Farah CS. Knowledge of oral cancer risk factors amongst high-risk Australians: findings from the LESIONS programme. Aus Dent J. 2016; 61(4):432-9.

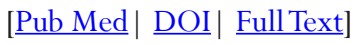

14. Reddy BS, Doshi D, Reddy MP, Kulkarni S, Gaffar A, Reddy VR. Oral cancer awareness and knowledge among dental patients in South India. J Cranio-Maxillofac Surg. 2012;40(6):521-4.[Pub Med | DOI | Full Text]

15. Rogers SN, Hunter R, Lowe D. Awareness of oral cancer in the Mersey region. Br J Oral Maxillofac Surg. 2011;49(3):176-81.[ [ Pub Med | DOI | Full Text]

16. Sudhir S, Sangle AR, Abhinav S, Juthikaa D. Awareness and knowledge of oral cancer in urban pune population: a survey based questionnaire study. Int J Clin Biomed Res. 2016;2(4):12-6.[Full Text] 